Check for updates

Cite this: RSC Adv., 2017, 7, 40005

Received 13th June 2017

Accepted 2nd August 2017

DOI: 10.1039/c7ra06617a

rsc.li/rsc-advances

\section{Multi-responsive, bidirectional, and large deformation bending actuators based on borax cross-linked polyvinyl alcohol derivative hydrogel $\uparrow$}

\author{
Liang Gao, (DD* Guoqiang Guo, Mengjuan Liu, Zeguo Tang, Liangxu Xie $\mathbb{D} \ddagger^{*}$ \\ and Yanping Huo (D)*
}

\begin{abstract}
This study demonstrates a multi-responsive high-performance bilayered hydrogel actuator (HA) obtained via coating a borax cross-linked $N^{1}, N^{1}$-diethylethane-1,2-diamine (DEEDA)-modified polyvinyl alcohol (PVA) microgel (PVA-DEEDA-borax) on a polyacrylic acid-coated biaxially oriented polypropylene (BOPP) substrate, and reveals that the amount of borax that is added plays a decisive role in the ultimate performance of the actuator. The HA is able to perform rapid, reversible, and sustainable bidirectional self-rolling deformation actuated by heat- and moisture-triggered swelling/deswelling of PVA-DEEDAborax gel, transforming into 2D/3D tube, wave and cross shapes through designing the assembly of actuators. The significant difference in the swelling behavior between PVA-DEEDA-borax and BOPP generates enough force to actuate the performance of the hydrogels as soft artificial muscle and selfadapted smart manipulator with capability of lifting masses much heavier than the actuator devices, showing potential applications in small-scale soft robotics.
\end{abstract}

\section{Introduction}

Polymeric actuators are being intensively explored due to their broad and significant applications in soft robotics, lab-on-achip, artificial muscle and biomimetic system. ${ }^{1}$ Among the huge variety of polymeric actuator materials, wet hydrogel has gained particular attention owing to its unique features that cannot be readily acquired from other material systems. ${ }^{2}$ Hydrogel has large chemical diversity, potentially offering multi-responsiveness to various stimuli and also adaptability for a large variety of applications. As mirrored in biomechanical processes, the hydrogel-based actuators (HAs) function based on the simple principle of volume change, making actuation behavior readily programmable and predictable. ${ }^{1 g}$ More attractively, a large number of hydrogels are biocompatible and biodegradable, which make them potential candidates for biomedical device applications. ${ }^{2}$ Given these advantages of

School of Chemical Engineering and Light Industry, Guangdong University of Technology, Guangzhou, China.E-mail: gaoliang@gdut.edu.cn; xlxhku@gmail.com; oryphuo@gdut.edu.cn

$\dagger$ Electronic supplementary information (ESI) available: Actuator modeling, performance optimization, structure characterization, and soft gripper. Demonstration (PDF): Movie S1-ESI: bending test for PVA, PVA-borax, PVA-DEEDA, and PVA-DEEDA-borax inside a refrigerator. (AVI), Movie S2-ESI: bending test for PVA, PVA-borax, PVA-DEEDA, and PVA-DEEDA-borax inside a hot oven. (AVI), Movie S3-ESI: transformation of the actuator as triggered by IR light. (AVI), Movie S4-ESI: artificial muscle demonstration. (AVI), Movie S5-ESI: smart manipulator demonstration (AVI). See DOI: 10.1039/c7ra06617a

\$ Present address: Chemistry Department, University of Hong Kong. hydrogels, HAs with programmable deformation, large bending force and fast actuation are intensively sought after for applications in various fields, such as robotics, ${ }^{3}$ biomedicine, ${ }^{4}$ microfluidics, ${ }^{5}$ and artificial muscles. ${ }^{6}$ A bilayered structure has been widely adopted for HAs. The bilayered system typically consists of an active layer and a passive layer. The isotropic dimensional changes in the active layer can be translated into the passive layer, thereby inducing the actuation behavior. The bilayered HA has a simple structure, making its motion predicable and controllable. ${ }^{7}$ However, numerous bilayered HAs synthesized previously suffer from several drawbacks, including unidirectional bending ${ }^{8}$ and absence of multi-responsive integration. ${ }^{9}$ Moreover, curing HAs that are able to form a tubular structure is still relatively rare. ${ }^{10}$

The abovementioned issues originate from the inadequate properties of hydrogel materials, because responsive bending of bilayered HAs theoretically relies on the intrinsic properties of active materials, such as mechanical modulus, and coefficient of thermal expansion and hygroscopic expansion (see Fig. S1 and supporting note 1 in ESI $\dagger$ ); $;^{7 b, 11}$ therefore, the type of hydrogels applied in HAs plays a decisive role in the ultimate performance of the actuator. To date, the most studied materials for bilayered HAs are cross-linked poly( $N$-isopropylacrylamide) (pNIPAM) microgel and its composite., ${ }^{\mathbf{9 1 1}, 12}$ pNIPAM microgel undergoes sol-to-gel phase transition as the temperature rises above the lower critical solution temperature (LCST). ${ }^{13}$ Several advanced fabrication processes have been employed on pNIPAM-based HAs to produce sophisticated nano-architecture, such as anisotropy $^{9 a}$ and nanostructured gradient, so that complex actuation 
with multi-dimensional structure transformation is achieved..$^{11,12}$ Although these pioneering efforts have demonstrated interesting actuation performance and applications, most of the reported pNIPAM-based HAs can only function in an aqueous environment (Table S1†). ${ }^{2}$ Accordingly, the device actuates only in response to temperature or $\mathrm{pH}$ and lacks integration of multiresponsiveness (Table $\mathrm{S} 1 \dagger$ ). Very recently, Serpe's group has reported that polyelectrolyte-modified pNIPAM-based HAs can perform actuation in "dry" state with large-degree curling. ${ }^{6}$ The fabrication requires the ordinal deposition of gold, poly(NIPAM)co-acrylic acid and poly(diallyldimethylammonium chloride) on a plastic substrate. Furthermore, the same group reported the shape memory properties of this device, and thorough investigations were made to gain insight into its bending behavior. ${ }^{14}$ In addition, developing HAs beyond pNIPAM has also been intensively studied. Sun's group has recently reported a highperformance poly(acrylic acid)/poly(allylamine hydrochloride)based walking device capable of rapid response to humidity. ${ }^{15}$ However, the humidity at which it can function ranges only from $11 \%$ to $40 \%$, which is narrower than that in many practical conditions. Furthermore, the bending angle cannot extend beyond $180^{\circ}$. The lack of study under higher humidity is likely due to the fact that the mechanical strength of the polyelectrolyte film dramatically decays as the degree of hydration increases. ${ }^{16}$ Zhang's group reported biocompatible bilayered HAs composed of layers of chitosan and cellulose/carboxymethylcellulose with opposite charges, ${ }^{17}$ but advanced transformations were merely created in response to the change in $\mathrm{pH}$. Overall, HAs show promising potentials for numerous significant applications but some challenging obstacles have to be overcome.

We have recently reported a post-synthetic modification of poly(vinyl alcohols) (PVA) with $N^{1}, N^{1}$-diethylethane-1,2-
diamine(DEEDA) (PVA-DEEDA) (Fig. 1a). ${ }^{18}$ The PVA-DEEDA series show highly tunable thermo- and $\mathrm{pH}$-responsiveness. As we have disclosed, when borax was added to $2.5 \mathrm{wt} \%$ PVADEEDA aqueous solution, irreversible gelation was instantly observed. The gelated PVA-DEEDA has good dimensional stability in both cold and hot water (Fig. S7a $\dagger$ ). ${ }^{18}$ However, here we noted that if borax is added to $1 \mathrm{wt} \%$ PVA-DEEDA solution at room temperature, a stable flowable aqueous solution (denoted as PVA-DEEDA-borax hereafter, Fig. 1a) is obtained (Fig. S7b $\dagger$ ). Upon heating, PVA-DEEDA-borax solution shows a stepwise phase transition (Fig. 1b): first, when the temperature was increased up to $40^{\circ} \mathrm{C}$, the PVA-DEEDA-borax solution exhibited LCST-type hydrophilic-to-hydrophobic phase transition, similar to PVA-DEEDA solution. Subsequently, when the temperature was further increased to $45^{\circ} \mathrm{C}$, sol-gel transition resulted in the formation of an opaque gel. This thermoresponsive gelation process is reversible, but a thermal hysteresis was observed (Fig. S8†). As we will discuss below, this sol-gel transition is necessary for the reversible expansion and contraction of hydrogel, otherwise, there is no actuation.

Herein, by taking advantages of the features of PVA-DEEDAborax microgel, namely its solution-processable feature and rapid change in dimension upon deswelling/swelling, ${ }^{18}$ we engineered a soft high-performance bilayered actuator made of PVA-DEEDA-borax and biaxially oriented polypropylene (BOPP). This PVA-DEEDA-borax-based HA is capable of responding to multi-stimuli, including moisture, temperature, and infrared light (photo-thermal). The PVA-DEEDA-borax layer is not only hygroexpansive but also thermocontractive. Therefore, changes in temperature and moisture collectively endow the HA with large $\left(360^{\circ}\right)$ reversible bidirectional bending actuation with curvature radii up to $3.14 \mathrm{~cm}^{-1}$, which is among a)

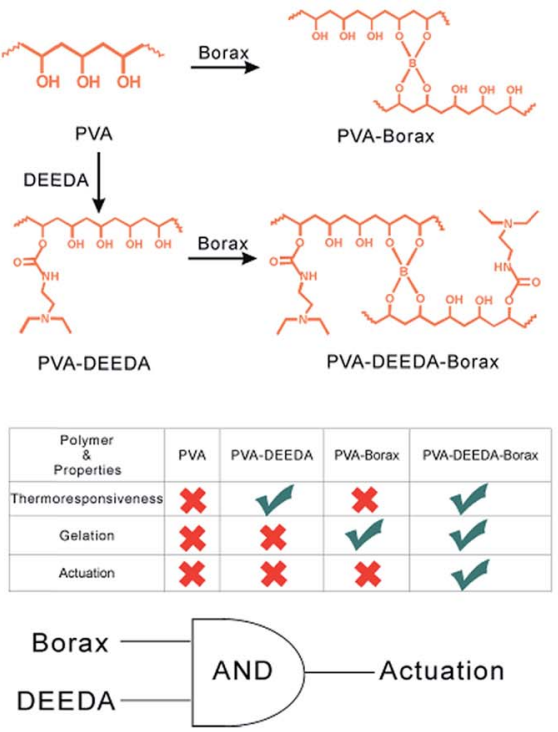

b)
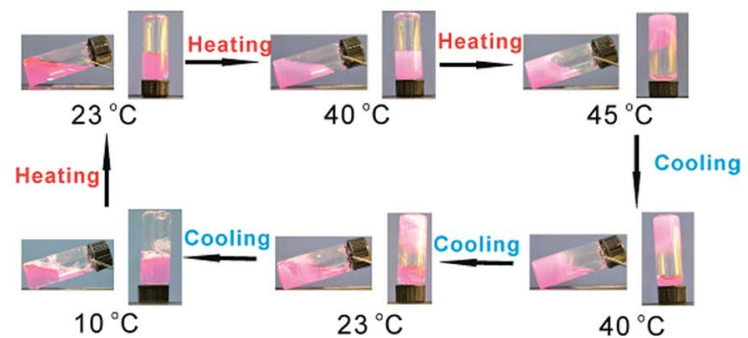

c)

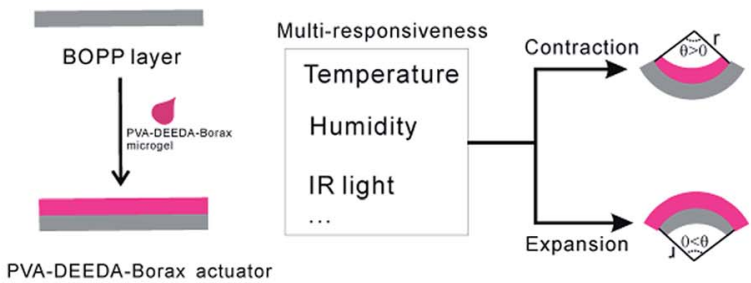

Fig. 1 (a) Schematic structure of PVA, PVA-borax, PVA-DEEDA, and PVA-DEEDA-borax, and their responsive properties. Both DEEDA functionalization and borax cross-link are necessary. (b) Gelation behavior of the PVA-DEEDA-borax upon heating (the solution was dyed by RhB to improve the visibility), while no such gelation can be observed for the non-cross-linked PVA-DEEDA-borax. (c) Fabrication of the bilayered actuator with PVA-DEEDA-borax as an active layer and the bidirectional bending in response to the change in temperature, humidity and photothermal effect. 
the largest curvature generated by state-of-the-art bilayered HAs (Table S1 $\dagger$ ). This HA can sustainably perform deformation in a wide temperature $\left(10-60{ }^{\circ} \mathrm{C}\right)$ and humidity (13-82\%) range. We discovered the co-existence of the borax cross-linked network and the DEEDA group, which are two indispensable contributors for the actuation (Fig. 1b and see discussion as below), suggesting the unique feature of the PVA-DEEDA-borax system. Various complex and advanced 2D/3D structure transformations such as letter shape, number shape and cross shape are successfully demonstrated on our HA. The actuator can work as an artificial muscle to rapidly $\left(0.6 \mathrm{~cm} \mathrm{~min}^{-1}\right)$ lift a mass 14 times the mass of the actuator as triggered by the simple changes in humidity. Finally, the use of our PVA-DEEDA-boraxbased actuator as a soft and self-adaptive manipulator is also demonstrated.

\section{Results and discussion}

\subsection{Actuator fabrication}

This actuator is fabricated by simply casting the PVA-DEEDAborax solution onto a BOPP substrate with a thickness of $c a$. $50 \mu \mathrm{m}$ (Fig. 1b). It is notable that the commercially available BOPP is coated with a layer of hydrophilic polyacrylic acid; therefore, the PVA-DEEDA borax solution can rapidly and uniformly spread on the BOPP substrate. To clarify whether the poly acrylic acid layer contributes to the actuation property, a control experiment is conducted using $\mathrm{O}_{2}$ plasma-etched BOPP without polyacrylic acid coating as a substrate. The actuators made of $\mathrm{O}_{2}$ plasma-etched BOPP and polyacrylic acid-coated BOPP show similar deformation behavior (Fig. S3 $\dagger$ ), suggesting that the layer of acrylic acid barely has an impact on the actuators, but it anchors PVA-DEEDA-borax on the BOPP substrate.

By adjusting the specific volume of the casting solution, the thickness of the active layer can be easily controlled over a wide range (Fig. S2 $\dagger$ ). Such type of controllable fabrication allows us to optimize the overall performance of the device since the thickness of the active layer intensively influences the actuation performance of bilayered HAs. ${ }^{7 b, 9 a}$ HAs with light weight $\left(10 \mathrm{mg} \mathrm{cm}^{-2}\right)$ and extra-large deformation are obtained with a PVA-DEEDAborax layer-to-BOPP thickness ratio of approximately 1.82 (see Fig. S3 and supporting note 2 in ESI $\dagger$ ). At this ratio, the layer of PVA-DEEDA-borax is highly uniform at a large scale (Fig. S4†).

\subsection{Bending of PVA-DEEDA-borax hydrogel-based actuator}

This actuator can rapidly bend to the direction of less expanded component, namely BOPP side at high temperature/low humidity. When the environment cools down and/or is humidified, our HA curls toward PVA-DEEDA-borax side (see Movies S1 and S2 $\dagger$ ). In contrast, no evident actuation is observed for PVA, PVA-DEEDA and PVA-borax (see Movies S1 and $\mathrm{S} 2 \dagger)$. These results clearly suggest that the combination of 3D network and thermoresponsive groups is indispensable for the actuation studied in this study, as illustrated in Fig. 1a. The 3D network ensures the sufficiently strong intermolecular interaction, and hence the expansion or shrinkage of layer materials can occur as a whole to trigger the macroscopic bending of the actuator. As we will discuss below, the thermoresponsive groups endow the dimensional shrinkage during heating. In principle, such a feature benefits the actuation (see discussion in the Actuator modeling section).

$\mathrm{X}$-ray diffraction (XRD) and attenuated total reflection (ATRFTIR) are used in this study. ATR-FTIR spectra for the PVADEEDA-borax layer suggest the formation of $\mathrm{B}-\mathrm{O}-\mathrm{C}$ bond due to the appearance of peaks at $1382 \mathrm{~cm}^{-1}$ and $1065 \mathrm{~cm}^{-1}$ (Fig. S9†). ${ }^{19}$ This confirms the occurrence of cross-linking between the hydroxyl groups of PVA-DEEDA and borate, as proposed in Fig. 1a. XRD of PVA-DEEDA-borax barely shows peaks of sodium borate crystal, further indicating that the borate is quantitatively consumed due to the cross-linking (Fig. S10†). Moreover, Differential Scanning Calorimetry (DSC) suggests increased temperature of glass transition upon addition of borax to PVA-DEEDA, implying decreased motion or/and rotation of PVA-DEEDA as a consequence of cross-linking (Fig. S11†). Overall, these results convincingly support that the PVA-DEEDA-borax layer of our HAs has three-dimensional cross-linked networks.

To characterize the bending behavior of the HAs in response to changes in temperature and humidity, we monitor the bending angle as a function of time. Temperature and humidity are controlled independently so that their contributions to actuation can be affirmed (Fig. S12 $\dagger$ ). As schematically illustrated in Fig. 2a, either temperature elevation or dehumidification can induce shrinkage of the PVA-DEEDA-borax layer, resulting in its curling at a positive angle. Indeed, our PVADEEDA-borax-based HA shows continuous bidirectional bending induced by the collective effect of temperature and humidity. For example, in Fig. 2b, we studied the humidity effect at four fixed temperatures. As either low temperature or high relative humidity favours water absorption of PVADEEDA-borax, under "wet and cold" conditions $(\mathrm{RH}=82.1 \%$ and $10{ }^{\circ} \mathrm{C}$ ), the PVA-DEEDA-borax layer expands and the HA strip bends toward the BOPP side. The absolute amount of bending increases linearly over the first $100 \mathrm{~min}$ and reaches maximum deformation of $-360^{\circ}$ after $120 \mathrm{~min}$. More promisingly, such large deformation can last for over $2 \mathrm{~h}$ without loosening, suggesting that our PVA-DEEDA-borax-based HA has good fatigue resistance, which also contributes to good recyclability of our HA devices (see below).

Moisture removal from the environment results in the deswelling of the PVA-DEEDA-borax layer. Therefore, at each given temperature, the PVA-DEEDA-borax layer gradually becomes less expanded as the humidity decreases. Under $\mathrm{RH}=13 \%$, the shrinkage of PVA-DEEDA-borax becomes overwhelming, and hence the HA bends toward the side of PVADEEDA-borax. On the other hand, owing to LCST-type thermoresponsive features of PVA-DEEDA-borax, ${ }^{18}$ changes in temperature can also independently control the bending of our HAs. For instance, under $\mathrm{RH}=82.1 \%$, when the temperature increases from $10{ }^{\circ} \mathrm{C}$ to $43{ }^{\circ} \mathrm{C}$, the maximum bending angle changes from $-360^{\circ}$ to $+70^{\circ}$. The similar trend of maximum bending angle $v s$. temperature is observed when the humidity is fixed at lower levels (Fig. S13†). 
a)
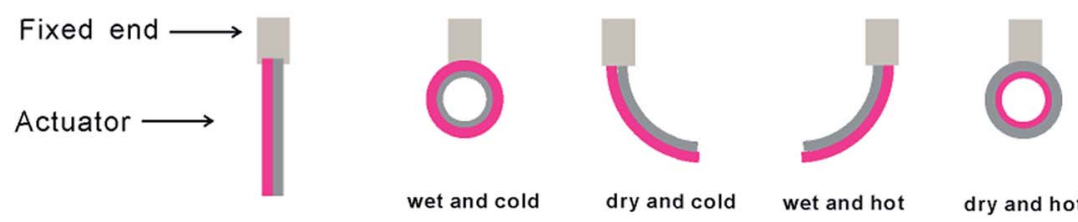

b)

C)
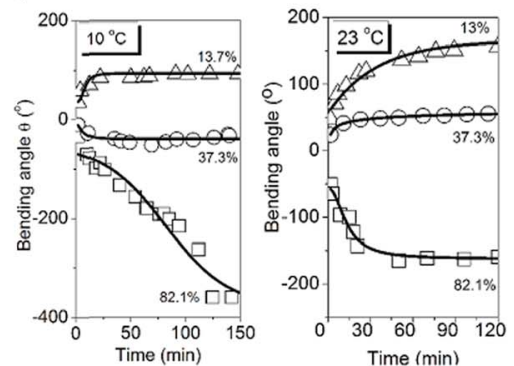

dry and cold

wet and hot

dry and hot

)
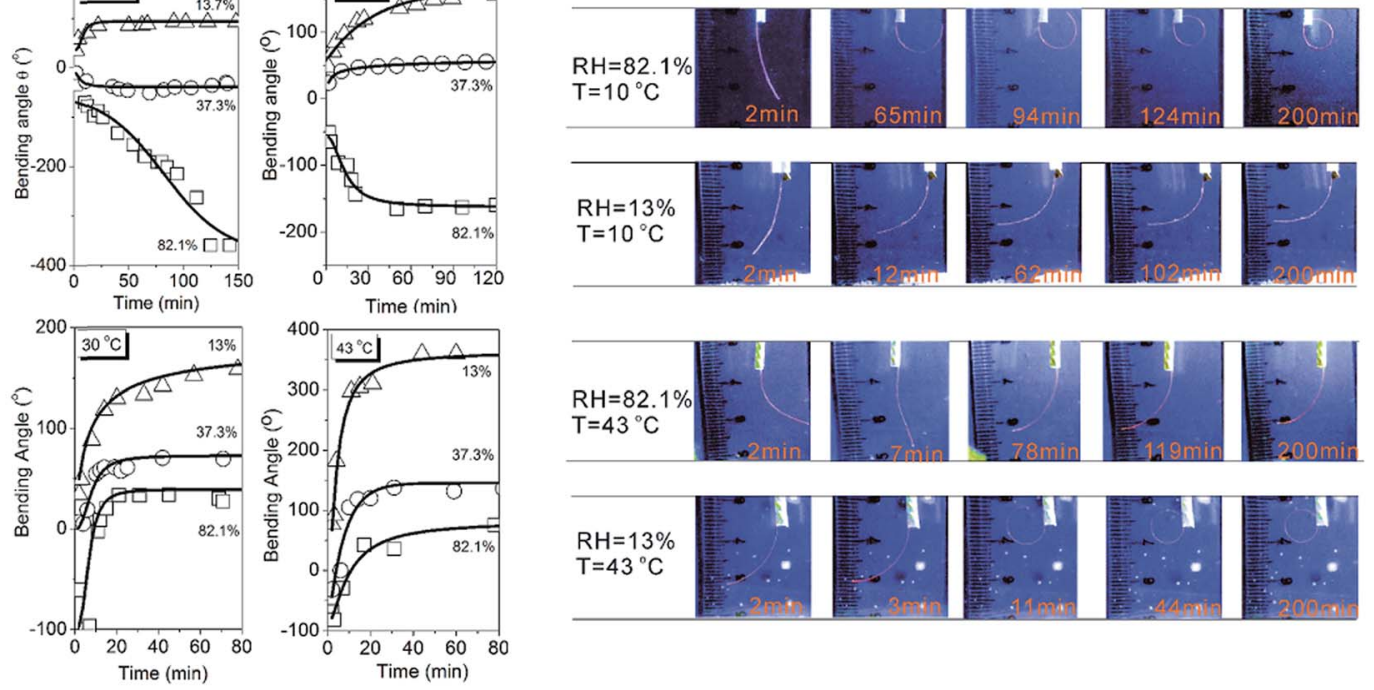

Fig. 2 (a) Schematic of four different bending behaviors in response to the temperature and humidity. (b) Bending kinetics and max bending angle of PVA-DEEDA-borax actuator. (c) Physical observation of the bending process of our HA under various conditions.

It is also noteworthy that the actuation kinetics of PVADEEDA-borax HAs is faster than that of several reported stateof-the-art HAs in "dry" state. ${ }^{6,20}$ The improved kinetics could be due to the expanded lattice of PVA-DEEDA-borax as evidenced by the low-angle shifting of XRD peak with comparison to non-cross-linked PVA-DEEDA (Fig. S10†). Such lattice expansion could be the consequence of charge repulsion of tetra-coordinated boron. Therefore, the free volume of PVADEEDA-borax matrix could be increased, accelerating humidification and dehumidification kinetics. Furthermore, the amount of borax added, which is directly related to the crosslink degree, dramatically influences both the maximum bending angle and the durability of bending (Fig. S5 and supporting note 2 in ESI $\dagger$ ). Notably, non-cross-linked PVA-DEEDA barely shows actuation (Fig. S6 $\dagger$ ). It is speculated that the 3D architecture offers elasticity of PVA-DEEDA-borax, and therefore the energy from external stimuli can be stored in the matrix to drive the motion of PVA-DEEDA-borax gel. On the other hand, HAs made of PVA-borax, which is supposed to have a similar 3D structure to that of PVA-DEEDA-borax, cannot actuate to perform the bending at all as summarized in Fig. 1a (Movies S1 and S2 $\dagger$ ). These observations suggest that the 3D architecture is necessary but not sufficient to guarantee the actuation. Several prerequisites, such as cross-link degree, charge densities of the polymer network, as well as cross-linked polymer concentration, have to be simultaneously satisfied so as to achieve adequate swelling and the elastic modulus for the actuation.
Since reasonable reversibility is desirable for practical applications, particularly for soft walking robotics and manipulators, we further study the reversibility of the actuator in response to the independent alternates of temperature and humidity. To evaluate the temperature-responsive reversible actuation, the environmental humidity is fixed at $\sim 48.3 \%$. We studied the deformation regularities of HA upon heating-cooling (Fig. 3a). At $10{ }^{\circ} \mathrm{C}$, the HA strip shows only slight bending with $c a$. $-20^{\circ}$. Temperature elevation causes our HA to bend toward the side of PVA-DEEDA-borax. When the temperature increases to $60{ }^{\circ} \mathrm{C}$, the HA curls up to a tube with a bending angle of $+360^{\circ}$. The bending gradually relaxes as the temperature decreases. When the temperature is decreased to $10^{\circ} \mathrm{C}$, the HA can almost return to its initial shape. Such heating/cooling cycles can be sustainably repeated for more than four times with only modest hysteresis. We also study the actuation reversibility in response to the humidity alternates (Fig. 3b). At a fixed temperature, the bending direction is immediately reversed as the humidity alternatively changes between $13 \%$ and $82.1 \%$. The alternative bending/unbending can be reproducible for several times without any significant decay in performance.

\subsection{Smart manipulator}

The above-demonstrated reversible actuation is further explored for complex structure transformation and small-scale manipulator. Although a large variety of bilayered HAs have been reported, many of the precedent devices can only show monotonous shape transformation. Integration of complex 
a)
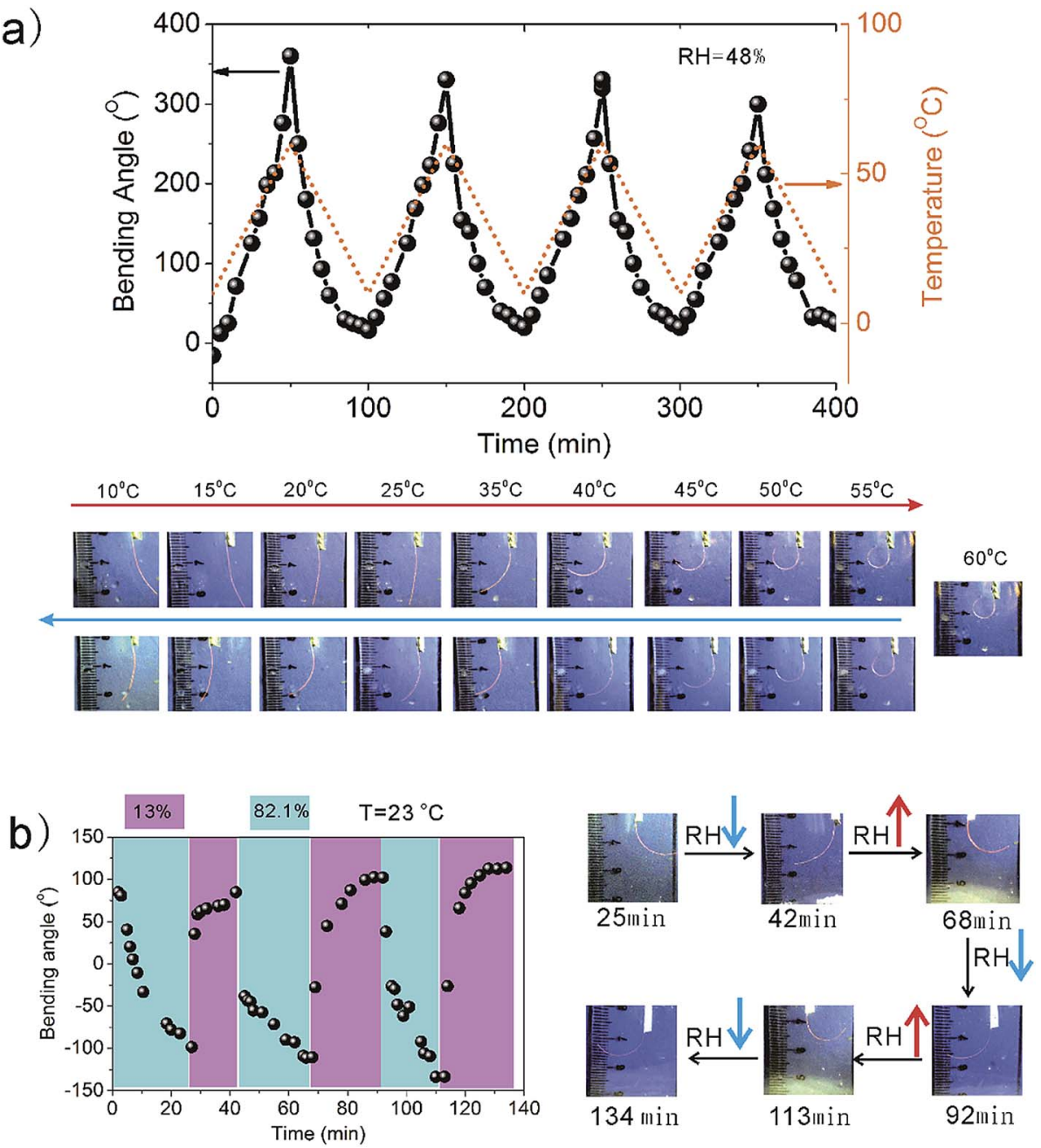

Fig. 3 Reversibility testing of the PVA-DEEDA-borax actuator: (a) repetitive temperature increase/decrease and (b) repetitive high (82.1\%)/low $(13 \%)$ humidity cycles of bending of a PVA-DEEDA-borax HA, showing good reversibility in response to the stimuli alternates.

transformation in a single HA device is challenging but essential for various applications. Owing to the mask-free and rapid fabrication process, it is easy to alter the design of the actuators so as to meet the practical requirements in terms of geometry, bending behavior, and force. Several previous examples demonstrate diverse and tunable gel shapes using hydrogelcarbon (graphene or carbon nanotube) nanocomposites as an active layer. ${ }^{3 b}$ However, doping carbon materials inevitably aggravates the cost and complexity of fabrication. Herein, we demonstrate that the assembly of PVA-DEEDA-borax HAs can actuate both 2D (Fig. 4a and b) and 3D (Fig. 4c and d) transformations. Fig. $4 \mathrm{a}$ and $\mathrm{b}$ illustrates a letter- and number-shaped structure programmed by changes in external moisture or/and temperature. In Fig. 4a, letter-shaped actuator device is fabricated via head-tail connection of three PVA-DEEDA-boraxbased HAs along the long axis. Explicitly, two actuators at the two ends of this device have the PVA-DEEDA-borax layer facing up, while the middle one has the PVA-DEEDA-borax layer facing down (Fig. 4a). Under ambient conditions $\left(23{ }^{\circ} \mathrm{C}\right.$ and $\left.30 \% \mathrm{RH}\right)$, this device initially takes on a $\mathrm{W}$ shape. When the temperature is decreased to $10^{\circ} \mathrm{C}$ and humidity increased to $60 \%$, the device is transformed from $\mathrm{W}$ shape into $\mathrm{M}$ shape. To further validate the advanced transformation in response to multi-stimuli, IRtriggered 2D number shape transformation was also demonstrated. Initially, the HAs are assembled into a number 3-shaped structure under ambient conditions $\left(23^{\circ} \mathrm{C}\right.$ and $\left.30 \% \mathrm{RH}\right)$. When IR light is applied, the number 3 -shaped actuator becomes a number 8-shaped actuator within $70 \mathrm{~s}$ (Movie S3†). Besides the above-mentioned 2D structure transformation, the ability to change the shape and control curvature in $3 \mathrm{D}$ structures is also conceptually demonstrated in our system. Literature reports on making reversible 3D structure translation out of HAs remain very scarce. One of the latest examples successfully achieved the 3D shape from flat films using liquid crystalline films. ${ }^{21}$ However, a very large temperature difference (e.g. $120^{\circ} \mathrm{C}$ ) is required to trigger the visible $3 \mathrm{D}$ actuation. We prove here that a simple cross-shaped assembly of our HA can be welded into 3D structures. In Fig. 4c, we first intentionally obtain a flat crossshaped device with both of their active layers facing down. In response to the changes in humidity, the four actuator strips simultaneously bend either downward or upward depending on the volume change in the PVA-DEEDA-borax layer. By simply 
a)

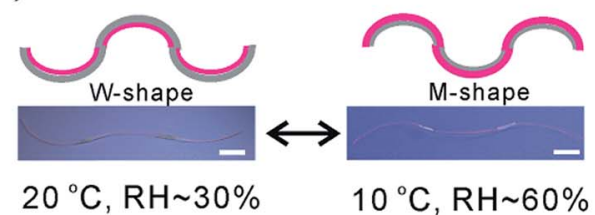

b)
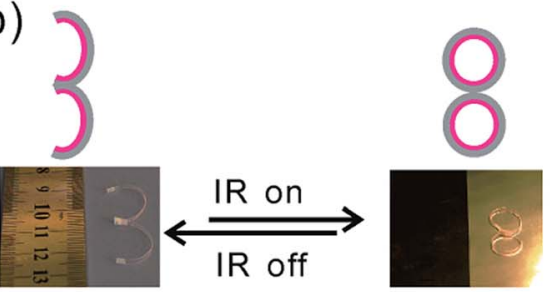

c) $\mathrm{T}=10^{\circ} \mathrm{C}$

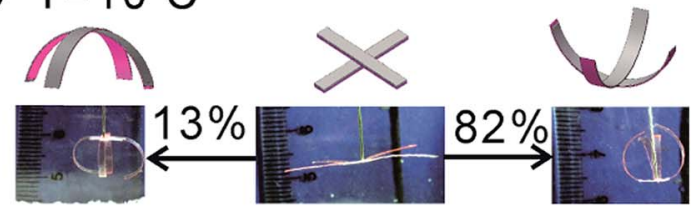

d) $\mathrm{RH}=82 \%$

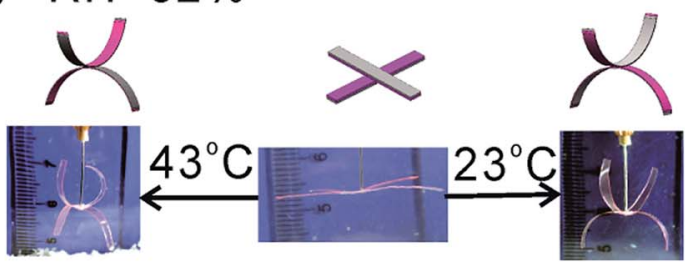

Fig. 4 Spatially programmable 2D/3D shape of PVA-DEEDA-borax-based HA: (a) W shape and $M$ shape as triggered by temperature and humidity; (b) numbers " 3 " and " 8 " shaped as triggered by IR light; spatial deformation: a cross made of two HA strips (c) with both active layers facing down; (d) with the active layer of one strip facing up and the other facing down.

alternating the sides of two of the actuators (Fig. 4d), bending can be achieved in the opposite direction. Notably, both of the above-mentioned $2 \mathrm{D}$ and $3 \mathrm{D}$ motions could be repeated upon the cycling of external stimulus.
We have demonstrated the capability of our HAs in conducting a large variety of mechanical motions in response to multi-stimulus; their practical applications were demonstrated by prototype "artificial muscle" and "smart manipulator".

a)
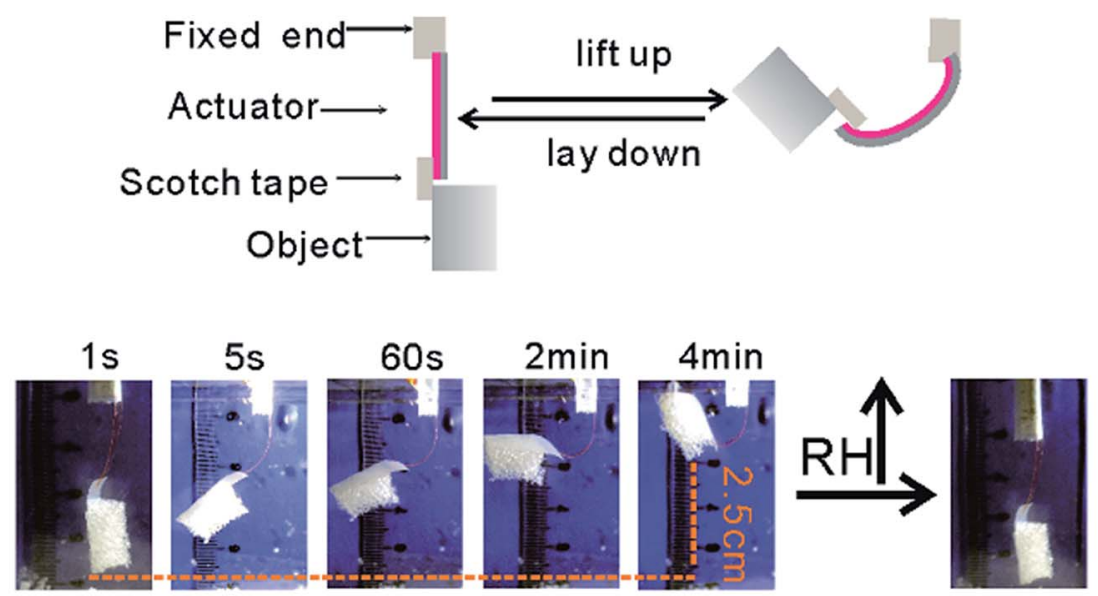

b)
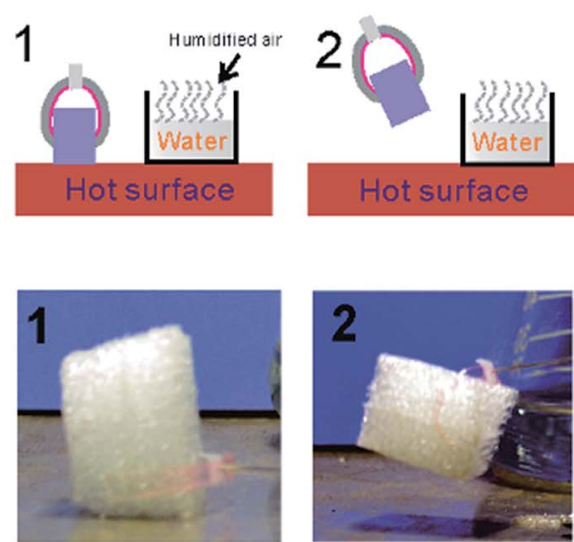

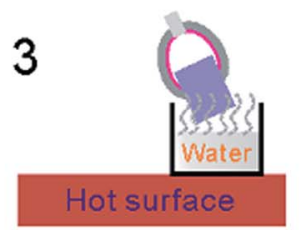

4

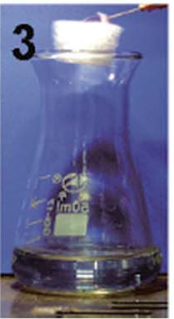

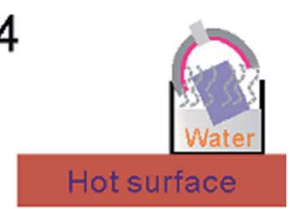

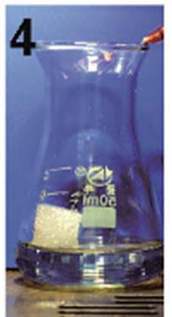

Fig. 5 (a) Photograph of artificial muscle based on PVA-DEEDA-borax HAs and (b) a two-finger gripper prototype used to demonstrate the ability to lift a light-weight object (a piece of polystyrene foam). 
Artificial muscle is of specific interest for the study of HA, as it can lift a mass much heavier than its own. ${ }^{6}$ As shown in Fig. 5a, at $60{ }^{\circ} \mathrm{C}$ and $\mathrm{RH}=13 \%$, our HA with a mass of $2 \mathrm{mg}$ can lift a mass of $23 \mathrm{mg}$ by $2 \mathrm{~cm}$ within $2 \mathrm{~min}$ (see Movie S4† and Fig. 5a). The loading capacity of the proposed artificial muscle is almost 12 times the mass of the device, similar to one of the reported state-of-the-art polymer-based muscles, ${ }^{6}$ but our HA shows much faster kinetics. When $\mathrm{RH}$ increases, the polystyrene foam hangs off the end of the device and the HA becomes almost straight again.

As a proof-of-concept of a smart manipulator, a two-finger gripper was constructed by one $4 \mathrm{~cm}$-long HA with the middle part fixed to a holder (Fig. 5b). This gripper was able to clamp, lift, and manipulate an object with weight $(0.2 \mathrm{~g})$ several times larger than its own $(0.04 \mathrm{~g})$ without the supply of electricity. Fig. 5b describes a self-adaptive four-step manipulation: first step, the 2-finger gripper gets close to the target object on a hot surface, its two "fingers" rapidly clamp onto the object due to the collective effect of temperature elevation and dehumidification is carried out; second step, the object is lifted off the hot surface; third step, the object gripped by HA is moved to the mouth of a flask which is filled with water (the long moving distance illustrates the strength of the clamping); and fourth step, since the humidity inside the flask is much higher than the hot surface, the gripper rapidly opens, dropping the polystyrene foam object (for the whole process, please see Movie S5 $\dagger$ ). The above-mentioned manipulation is repetitive and can manipulate objects with different shapes (Movie S5†).

\subsection{Actuator modeling}

To gain insight into the features responsible for the actuation, the mechanical behavior and coefficient of thermal expansion are compared between PVA-DEEDA-borax and PVA-borax. As demonstrated earlier in this study, the actuator based on the PVA-borax can actuate in response to temperature and humidity, while the other cannot. Therefore, this comparison allows us to deduce the critical properties that are responsible for the observed actuation.

According to literature, ${ }^{7}$ the linear elastic theory of beams ${ }^{23}$ (see details in ESI $\dagger$ ) is applicable to the bilayered actuator made of thermoexpansive and hygroexpansive materials. Therefore, eqn (1) is used to estimate the curvature of the bilayered actuator (see Fig. 6):

$$
\begin{aligned}
& k \propto\left(\alpha_{2}-\alpha_{1}\right) \Delta T+\beta_{2} \Delta C_{2}-\beta_{1} \Delta C_{1}= \\
&\left(137.5 \times 10^{-6}-\alpha_{1}\right) \Delta T-\beta_{1} \Delta C_{1}
\end{aligned}
$$

$\beta_{1}$ is the coefficient of hygroscopic expansion (CHE) of PVADEEDA-borax (here, the hygroscopicity of BOPP is negligible, and therefore, it decays to zero); $\Delta C_{1}$ is the change in moisture concentration in PVA-DEEDA-borax as a function of temperature, which is a negative value; $\Delta T$ is the temperature change; $\alpha_{1}$ and $\alpha_{2}$ are the coefficients of thermal expansion (CTE) for the PVA-DEEDA-borax and BOPP films, respectively. Clearly, $\alpha_{1}$ should be negative due to the deswelling during thermogelation, ${ }^{18}$ while $\alpha_{1}$ should be positive. ${ }^{10}$
Based on eqn (1), CTE and CHE of layer materials are clearly two critical parameters that govern the heat- and humidityinduced bending of bilayered HAs. Therefore, we concentrate on the comparison of CTE and CHE between PVA-DEEDAborax and PVA-borax. As the CTE is typically very small, usually in the magnitude of $10^{-4}-10^{-6}$ per $K$, its quantification is difficult using experimental techniques. ${ }^{7,25}$ Therefore, we perform molecular dynamic simulation to evaluate the CTE of PVA-DEEDA-borax and PVA-borax gel. Fig. 7c and d shows volume changes and the corresponding snapshot of molecular conformation at high and low temperatures. A similar simulation has been applied to calculate the CTE of soft materials (Table 1). ${ }^{26}$

Based on our molecular dynamic results, the CTE values for PVA-DEEDA-borax and PVA-borax are about $-3.26 \times 10^{-4}$ and $1.5 \times 10^{-3}$. Therefore, during heating, the PVA-DEEDA-borax layer material shrinks, while the PVA-borax layer material experiences considerable expansion. We further test the moisture content of PVA-DEEDA-borax or PVA-borax against the temperature change (see Fig. 7c) using thermogravimetric analysis (TGA). Clearly, the moisture content of PVA-DEEDAborax is more sensitive to the temperature than that of PVAborax, suggesting a larger $\Delta C$. The stronger temperature dependence can be explained by the enhanced hydrophobicity as a result of thermally induced phase transition.

Even though these modeling parameters cannot exactly fit our experimental data due to their simplicity, the current study on material properties can clearly explain the observed differences in actuation between PVA-borax-based and PVA-DEEDAborax-based HAs. When combined with the positive CTE and negative CHE of the BOPP layer, it can be concluded that the
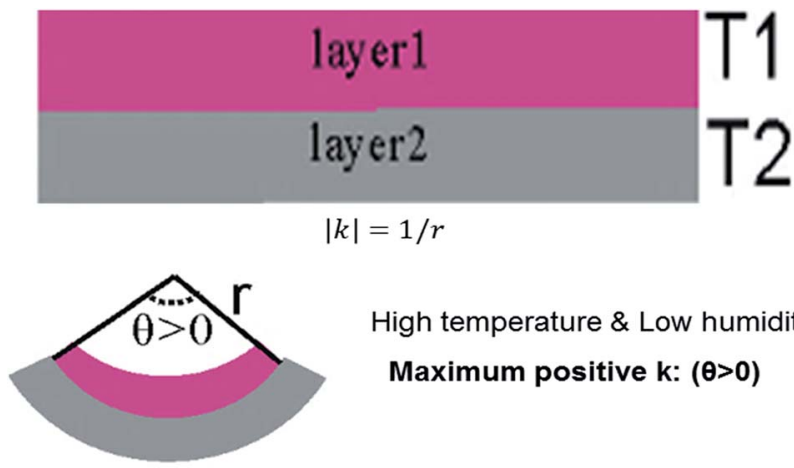

High temperature \& Low humidity

Maximum positive $\mathrm{k}:(\theta>0)$

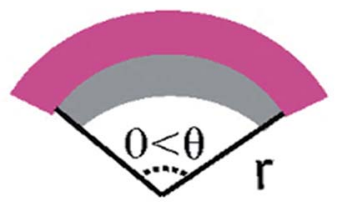

Low temperature \& High humidity

Maximum negative $\mathrm{k}:(\theta<0)$

Fig. 6 Schematic of the bilayered PVA-DEEDA-borax hydrogel actuator. Pink layer stands for PVA-DEEDA-borax, while gray layer stands for BOPP substrate. To achieve maximum bending actuation, a layer 1 material should have a high $\mathrm{CHE}$ and a low or even negative CTE. 
a)
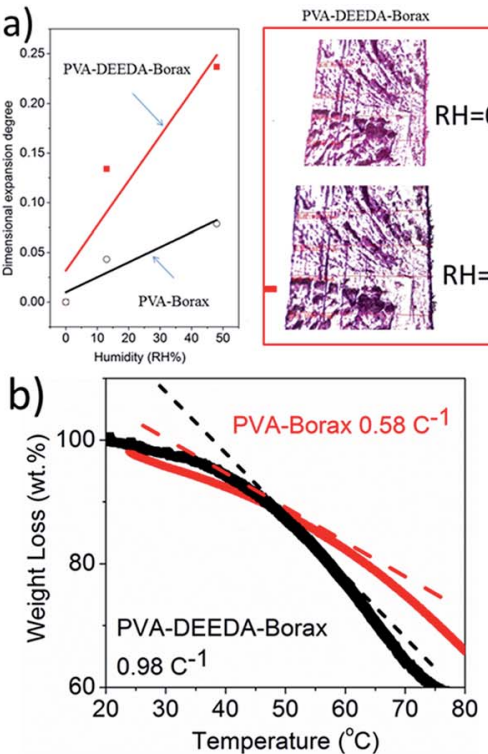

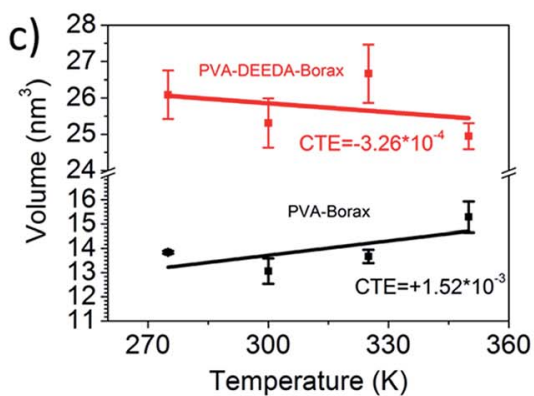

d)

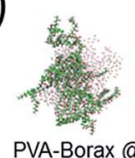

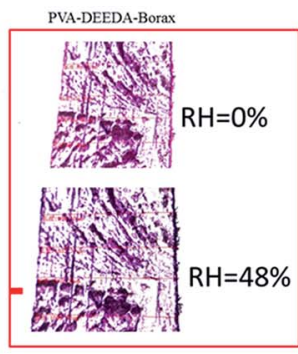

\section{(1)}

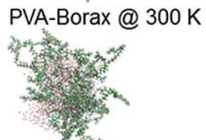

PVA-DEEDA-Borax @ $300 \mathrm{~K}$ PVA-DEEDA-Borax@ $350 \mathrm{~K}$

Fig. 7 (a) CHE as determined by monitoring the dimensional changes with humidity. (b) Water content as a function of temperature gradient. (c) CTE using molecular dynamic simulation as determined by calculating the volume changes along with the changes in temperature. (d) Molecular dynamic conformation at different temperatures.

Table $1 \mathrm{CTE}, \mathrm{CHE}$, and $\Delta \mathrm{C}$ of different layer materials

\begin{tabular}{|c|c|c|c|}
\hline \multirow[b]{2}{*}{ Sample } & \multicolumn{3}{|l|}{ Parameter } \\
\hline & CTE $(\alpha), \mathrm{K}^{-1}$ & $\operatorname{CHE}(\beta), \% \mathrm{RH}^{-1}$ & $\Delta C, \mathrm{C}^{-1}$ \\
\hline ВOPР & $\begin{array}{l}1.375 \times 10^{-4} \mathrm{~K}^{-1} \\
\text { (ref. 24) }\end{array}$ & $\sim 0$ & $\sim 0$ \\
\hline PVA-DEEDA-borax & $-3.26 \times 10^{-4 a}$ & $\sim 4.9 \times 10^{-3 b}$ & $\sim-0.98^{c}$ \\
\hline PVA-borax & $1.52 \times 10^{-3 a}$ & $\sim 1.3 \times 10^{-3 b}$ & $\sim-0.58^{c}$ \\
\hline \multicolumn{4}{|c|}{$\begin{array}{l}{ }^{a} \text { Calculated values based on molecular dynamic simulation. } \\
{ }^{b} \text { Measured using an optical microscope to track the dimensional } \\
\text { changes of hydrogel after equilibrating at varying humidity. } \\
{ }^{c} \text { Measured by TGA. The samples are equilibrated under } 50 \% \mathrm{RH} \\
\text { before testing. }\end{array}$} \\
\hline
\end{tabular}

positive CTE value of PVA-borax would compromise the bending of bilayered HAs, while the negative CTE of PVADEEDA-borax can make the bending more pronounced. More interestingly, the CHE of PVA-DEEDA is also larger than that of PVA-borax. This could be due to the increased free volume of PVA-DEEDA-borax as a result of the disruption of hydrogen bonding in PVA network; $;^{16,26,27}$ water can more readily swell PVA-DEEDA-borax.

\section{Conclusions}

In conclusion, we demonstrate that a hydrogel actuator fabricated through casting borax cross-linked PVA-DEEDA (PVADEEDA-borax) on the BOPP substrate is able to actuate performing structure transformation and manipulate objectives in response to temperature and humidity. The obtained HA exhibits an ultra large reversible bidirectional bending $\left(-360^{\circ}\right.$ to $+360^{\circ}$ ), with a curvature as large as $3.14 \mathrm{~cm}^{-1}$, and complex
2D and 3D transformations in response to humidity, temperature, and IR light. Furthermore, the HA can work as an artificial muscle and manipulator to lift a mass over 10 times the mass of the actuator within seconds. We believe that the actuation of PVA-DEEDA-borax-based HA can be further developed by incorporating additional responsiveness, such as magnetite. In addition, by taking advantage of the biological compatibility of PVA backbone, bio-related applications can also be further explored. When compared with the PVA-borax-based actuator, which barely exhibits actuation, the high-performance actuation of the PVA-DEEDA-borax-based actuator is dedicatedly related to high CHE, low CTE and high $\Delta C$ of the PVA-DEEDAborax layer material.

\section{Experimental}

\subsection{Synthesis of PVA-DEEDA and PVA-DEEDA-borax materials}

Polyvinyl alcohol (PVA, Aldrich, average $M_{\mathrm{w}}=16600$ (approximately 380 vinyl alcohol monomer units), hydrolysis degree $=99 \%$ ), $N^{1}, N^{1}$-diethylethane-1,2-diamine (DEEDA, Acros, purity $>98 \%$ ), carbonyldiimidazole (CDI, Dikemann (China), purity $>$ 99\%), dry DMSO (Dikmann (China), purity > 99\%), and acetone (ACS, AR grade) are used as received. All aqueous solutions are prepared using Milli-Q 18 M DI water. PVADEEDA with a degree of functionalization of approximately $23 \%$ is synthesized according to our previous report, ${ }^{\mathbf{1 8}}$ where the degree of DEEDA functionalization increases from $20 \%$ to $23 \%$ by simply increasing the reaction time from $90 \mathrm{~h}$ to $100 \mathrm{~h}$.

\subsection{Fabrication of actuators}

The actuators are fabricated by the solution casting method. The pre-gel solution of PVA-DEEDA-borax is prepared by 
adding $25 \mu \mathrm{L} 4 \mathrm{wt} \%$ borax solution to $500 \mu \mathrm{L} 1 \mathrm{wt} \%$ PVA-DEEDA solution. After stirring at $10{ }^{\circ} \mathrm{C}$ for $5 \mathrm{~min}$, the pre-gel solution is casted on a commercial polyacrylic acid-coated BOPP tape with a thickness of $50 \mu \mathrm{m}$. Then, the PVA-DEEDA-borax solution is evaporated in an oven at $50{ }^{\circ} \mathrm{C}$ for $30 \mathrm{~min}$. Subsequently, the obtained bilayered actuator is cut into strips of size $0.2 \mathrm{~cm} \times$ $2 \mathrm{~cm}$ and stored in a chamber with humidity of $30 \%$ and a temperature of $10{ }^{\circ} \mathrm{C}$ for further characterization. After a series of optimization experiments, we identified that casting $105 \mu \mathrm{L} \mathrm{cm}{ }^{-2}$ PVA-DEEDA-borax, which contains $10 \mathrm{wt} \%$ borax, formed an actuator with $\pm 360^{\circ} \mathrm{C}$ bending ability as a function of both temperature and humidity.

\subsection{Humidity, temperature, and IR-driven test}

The relative humidity is controlled using a transparent chamber half filled with a slushy mixture of salt and DI water (Fig. S11 ). The chamber is equipped with a humidity sensor and a thermocouple (Fig. S11†). Similar to the previous study, ${ }^{22}$ we observed that the humidity can be maintained with very small fluctuation in a wide temperature range. The whole chamber is put into an oven for temperature control. The bending of the actuator is recorded using a digital camera and analyzed using the image J software. The light source is a commercial infrared light $(200 \mathrm{~W})$, and the distance was $c a .30 \mathrm{~cm}$ from the sample to the light source.

\subsection{Physical characterization}

The bending curvatures of the actuator, all optical images, and videos were captured using a digital camera (Nikon, D5400). PXRDs were obtained on a Riguku D/Max-2400 X-ray diffractometer with a Cu sealed tube $(\lambda=1.54178 \AA)$. UV-Vis spectroscopic measurements were recorded on a Hewlett-Packard 8453 spectrophotometer coupled with a circulator temperature controller. To achieve thermal equilibrium, the samples were kept at a set temperature for at least $10 \mathrm{~min}$. The thermally responsive soluble-insoluble phase transition of the PVADEEDA-borax aqueous solution was characterized by measuring the percent transmittance $(\% T)$ at $\lambda=500 \mathrm{~nm}$. Optical microscopy (OD500M, Sunny Optical Technology Co., Ltd China) is used to image the cross-section of actuators. The surface structures of the hydrogels were further characterized using attenuated total reflection Fourier transform infrared spectroscopy (ATR-FTIR, NICOLET 5700, Thermo Nicolet). DSC was recorded on a NETZSCH $204 \mathrm{~F} 1$ at a ramping rate of $10{ }^{\circ} \mathrm{C} \min ^{-1}$ from $10{ }^{\circ} \mathrm{C}$ to $120{ }^{\circ} \mathrm{C}$ under $\mathrm{N}_{2}$ flow. To remove the thermal history, all samples were preheated to $120{ }^{\circ} \mathrm{C}$ and then cooled down to $10{ }^{\circ} \mathrm{C}$.

\section{Author contributions}

Dr Liang Gao conceived the idea, performed the experiment, and wrote the paper. All authors have given approval to the final version of the manuscript.

\section{Funding sources}

We are grateful for financial support from NSFC (Grant No. 51603046, 61671162), "One-hundred Young Talents of Guangdong University of Technology (class A)" program” (Grant 220413132) and Technology Planning Project of Guangdong Province (Grant No. 2016A010103031).

\section{Conflicts of interest}

There are no conflicts to declare.

\section{Acknowledgements}

Dr Liang Gao thanks Dr Tao Yu at Sun Yatsen University for DSC testing.

\section{References}

1 (a) J. A. Lv, Y. Liu, J. Wei, E. Chen, L. Qin and Y. Yu, Nature, 2016, 537, 179; (b) E. W. H. Jager, E. Smela and O. Inganäs, Microfabricating conjugated polymer actuators, Science, 2000, 290, 1540; (c) M. Ma, L. Guo, D. G. Anderson and R. Langer, Science, 2013, 339, 186; (d) A. Lendlein, H. Jiang, O. Junger and R. Langer, Nature, 2005, 434, 879; (e) P. Fratzl and F. G. Barth, Nature, 2009, 462, 442; $(f)$ L. Hines, K. Petersen, G. Z. Lum and M. Sitti, Adv. Mater., 2016, 1603483; $(g)$ L. Ionov, Biomimetic Hydrogel-Based Actuating Systems, Adv. Funct. Mater., 2013, 23, 4555; (h) L. Ionov, Langmuir, 2015, 31, 5015; (i) H. Arazoe, D. Miyajima, K. Akaike, F. Araoka, E. Sato, T. Hikima, M. Kawamoto and T. Aida, Nat. Mater., 2016, 15, 1084; (j) E. Palleau, D. Morales, M. D. Dickey and O. D. Velev, Nat. Commun., 2013, 4, 2257; ( $k$ ) Q. Zhao, J. W. C. Dunlop, X. Qiu, F. Huang, Z. Zhang, J. Heyda, J. Dzubiella, M. Antonietti and J. Yuan, Nat. Commun., 2014, 5, 4293; (l) H. Yuk, S. Lin, C. Ma, M. Takaffoli, N. X. Fang and X. Zhao, Nat. Commun., 2017, 8, 14230; $(\mathrm{m})$ Y. Osada, H. Okuzaki and H. Hori, Nature, 1992, 355, 242.

2 (a) L. Ionov, Mater. Today, 2014, 17, 494; (b) M. Wei, Y. Gao, X. Li and M. J. Serpe, Polym. Chem., 2017, 8, 127.

3 (a) W. J. Zheng, N. An, J. H. Yang, J. Zhou and Y. M. Chen, ACS Appl. Mater. Interfaces, 2015, 7, 1758; (b) E. Wang, M. S. Desai and S. W. Lee, Nano Lett., 2013, 13, 2826.

4 (a) A. Pelah, R. Seemann and T. M. Jovin, J. Am. Chem. Soc., 2007, 129, 468; (b) X. Zhao, J. Kim, C. A. Cezar, N. Huebsch, K. Lee, K. Bouhadir and D. J. Mooney, Proc. Natl. Acad. Sci. U. S. A., 2011, 108, 67.

5 S. R. Sershen, G. A. Mensing, M. Ng. N. J. Halas, D. J. Beebe and J. L. West, Adv. Mater., 2005, 17, 1366.

6 M. R. Islam, X. Li, K. Smyth and M. J. Serpe, Angew. Chem., Int. Ed., 2013, 52, 10330.

7 (a) P. Chen, S. He, Y. Xu, X. Sun and H. Peng, Adv. Mater., 2015, 27, 4982; (b) M. Amjadi and M. Sitti, HighPerformance Multiresponsive Paper Actuators, ACS Nano, 2016, 10, 10202. 
8 M. Weng, P. Zhou, L. Chen, L. Zhang, W. Zhang, Z. Huang, C. Liu and S. Fan, Adv. Funct. Mater., 2016, 26, 7244.

9 (a) L. Liu, A. Ghaemi, S. Gekle and S. Agarwal, Adv. Mater., 2016, 28, 9792; (b) Y. Hu, J. S. Kahn, W. Guo, F. Huang, M. Fadeev, D. Harries and I. Willner, J. Am. Chem. Soc., 2016, 138, 16112.

10 L. Chen, M. Weng, Z. Zhou, Y. Zhou, L. Zhang, J. Li, Z. Huang, W. Zhang, C. Liu and S. Fan, ACS Nano, 2015, 9, 12189.

11 C. Yao, Z. Liu, C. Yang, W. Wang, X. J. Ju, R. Xie and L. Y. Chu, Adv. Funct. Mater., 2015, 25, 2980.

12 (a) T. A. Asoh, M. Matsusaki, T. Kaneko and M. Akashi, Adv. Mater., 2008, 20, 2080; (b) J. Kim, J. A. Hanna, R. C. Hayward and C. D. Santangelo, Thermally responsive rolling of thin gel strips with discrete variations in swelling, Soft Matter, 2012, 8, 2375; (c) J. Kim, J. A. Hanna, M. Byun, C. D. Santangelo and R. C. Hayward, Science, 2012, 335, 1201; (d) Z. Hu, X. Zhang and Y. Li, Science, 1995, 269, 525; (e) D. Kim, H. Kim, E. Lee, K. S. Jin and J. Yoon, Chem. Mater., 2016, 28, 8807.

13 Y. Zhao, Y. Cao, Y. Yang and C. Wu, Macromolecules, 2003, 36, 855-859.

14 X. Li and M. J. Serpe, Adv. Funct. Mater., 2016, 26, 3282.

15 Y. Ma, Y. Zhang, B. Wu, W. Sun, Z. Li and J. Sun, Angew. Chem., Int. Ed., 2011, 50, 6254.
16 L. Gao, T. Kong, G. Guo and Y. Huo, Int. J. Hydrogen Energy, 2016, 41, 20373.

17 J. Duan, X. Liang, K. Zhu, J. Guo and L. Zhang, Soft Matter, 2017, 13, 345.

18 L. Gao, T. Kong and Y. Huo, Macromolecules, 2016, 49, 7478. 19 S. Spoljaric, A. Salminen, N. D. Luong and J. Seppälä, Eur. Polym. J., 2014, 56, 105.

20 B. P. Lee and S. Konst, Adv. Mater., 2014, 26, 3415.

21 Y. Yang, Z. Pei, Z. Li, Y. Wei and Y. Ji, J. Am. Chem. Soc., 2016, 138, 2118.

22 J. F. Young, Humidity control in the laboratory using salt solutions-a review, J. Appl. Chem., 1967, 17, 241.

23 C. Wen-Hwa, M. Mehregany and R. L. Mullen, J. Micromech. Microeng., 1993, 3, 4.

24 M. M. Hamedi, V. E. Campbell, P. Rothemund, F. Güder, D. C. Christodouleas, J. F. Bloch and G. M. Whitesides, Adv. Funct. Mater., 2016, 26, 2446-2453.

25 C. Dudescu, A. Botean and M. Hardau, Mater. Plast., 2013, 50, 55-59.

26 C. Wei, D. Srivastava and K. Cho, Nano Lett., 2002, 2(6), 647650.

27 H. E. Assender and A. H. Windle, Crystallnity in polyvinyl alcohol 1. An X-ray diffranction study of atactic PVOH, Polymer, 1998, 39, 4295-4302. 